\title{
POSITIVE INFLUENCE OF AMINOSILANES ON ANTI-EPCAM ANTIBODY IMMOBILIZATION ON A GLASS SURFACE
}

\author{
Paula Kamińska ${ }^{1}$, Karolina Buszka ${ }^{1,2}$, Przemysław Pietras ${ }^{3}$, Maciej Zabel ${ }^{4}$, Michał Nowicki ${ }^{1}$, \\ Joanna Budna-Tukan ${ }^{1}$
}

\begin{abstract}
Immobilization of antibodies has a number of promising applications, including detection of biomolecules and cells. Well-oriented antibodies are required to bind them effectively. To eliminate the problem of random antibodies' orientation, the surface of the device can be modified with silanes. This study aimed at elucidating if selected aminosilanes were able to bind antibodies in the appropriate orientation and thus retain their binding activity. Silanization of glass slides was performed using three amino-functional trialkoxysilanes - A, AE, and AEE. The immunofluorescent reaction was used to evaluate the potential of the silanized glass surface to bind anti-EpCAM antibodies. The affinity of selected anti-EpCAM HEA125 antibodies labeled with fluorochrome to tested silanized surfaces was evaluated by measuring the mean fluorescence intensity (MFI) in each analyzed area. The presented silanes effectively bound antibodies. Higher fluorescence intensity was noticed in the case of silane-coated glass slides in comparison to unmodified ones. The differences in the contact angles also confirmed this result. In the case of silane A, the fluorescence intensity reflected the amount of bound antibodies. However, there was no such a relation in the case of the silanes AE and AEE. Although our research gave promising results, the usefulness of selected silanes needs to be confirmed by further studies using cancer cells.
\end{abstract}

Running title: Aminosilanes as enhancers of antibody immobilization

Keywords: aminosilanes, silanization, antibody immobilization, anti-EpCAM antibody, immunofluorescence, contact angle measurement

\footnotetext{
${ }^{1}$ Department of Histology and Embryology, Poznań University of Medical Sciences, Poznań, Poland

${ }^{2}$ Faculty of Agronomy, Horticulture and Bioengineering, Poznań University of Life Sciences, Poznań, Poland

${ }^{3}$ Adam Mickiewicz University Foundation, Poznań Science and Technology Park, Poznań, Poland

${ }^{4}$ Department of Anatomy and Histology, Collegium Medicum, University of Zielona Góra, Zielona Góra, Poland

*Correspondence: jbudna@ump.edu.pl

Full list of author information is available at the end of article
} 


\section{Introduction}

In the last years, the analysis of rare cells has started to be used for diagnostic purposes. An example of such cells are circulating tumor cells (CTCs). These cells originate from the primary tumor, and after detachment, circulate in the patient's bloodstream [1,2]. Only a few CTCs can be found per 10 million leukocytes in $1 \mathrm{~mL}$ of blood [3]. Their analysis can be extremely useful, among others, in the rapid detection of tumors [4]. CTCs in the tested blood sample are an indicator of cancer spread and can be correlated with the stage of the disease [59]. Early diagnostics based on CTC detection can be beneficial for fast selection of an appropriate treatment, monitoring its effectiveness, and detecting residual disease [10]. All this significantly increases the chances of patients' survival.

Currently, there are many methods for the detection of CTCs, which rely both on in vitro and in vivo analysis [8,11,12]. Detection technologies can be based on the analysis of tumor-specific surface markers [13] or the physical parameters of CTCs, such as size, formability, and density [14]. Marker-based methods most often use the epithelial cell adhesion molecule (EpCAM) antigen, a transmembrane protein highly expressed in many types of cancer, not present on the normal blood cells [15]. Among in vitro technologies based on EpCAM CellSearch ${ }^{\circledR}$, AdnaTest or PCR analysis are most frequent [1].

Worth mentioning is the CellCollector ${ }^{\circledR}$ device, which is the first in vivo commercial test in the world to detect CTCs directly in the patient's bloodstream [8]. The indisputable advantage of in vivo over in vitro methods is a much higher blood test volume (1 L vs. $10 \mathrm{~mL}$, respectively), which is invaluable in rare events such as CTCs [12]. To create such a solid phase-based CTC detection device, it is necessary to immobilize the antibodies on its surface. In order to do this, the carrier must be covered with an intermediate layer, interacting with the antibodies by the formation of covalent bonds or through bioaffinity interaction. Such chemical or topographic modifications of solid phase carriers allow them to bind antibodies more efficiently and achieve higher yields of captured cells [16].

In recent years, compounds based on silicon have been used. Biosensors such as silica-coated nanoparticles are sensitive and selective technologies [17]. Biosensing and diagnostic platforms with high sensitivity, specificity, and fast response time are based on immobilized biomolecules such as antibodies, aptamers, enzymes, nucleic acids, receptors, or whole cells to detect target analytes [18]. Silanization is commonly used to introduce surface functional groups to silica and glass surfaces [19]. The majority of silica surface modification methods is based on the concentration reaction be- tween surface $\mathrm{Si}-\mathrm{OH}$ groups and acyloxy-, chloro-, or alkoxysilanes leading to the formation of a stable bond with the surface and release of byproduct [20]. Antibodies can be immobilized on the modified surface by forming covalent bondage between their functional group and the complementary coupling group of silanes [21]. This study aimed at elucidating if selected aminosilanes were able to bind antibodies in the appropriate orientation and thus retain their binding activity.

\section{Materials and methods \\ Preparation of silanized glass surfaces}

Silanization of glass slides was performed using three amino-functional trialkoxysilanes: 3-Aminopropyltriethoxysilane - A (> 99\%, Sigma-Aldrich, St. Louis, MO, USA), [3-(2-Aminoethylamino)propyl] trimethoxysilane - AE (AE 97\%, Sigma-Aldrich, St. Louis, MO, USA) and (3-Trimethoxysilylpropyl) diethylenetriamine - AEE (AEE 85\%, ABCR GmbH, Karlsruhe, Germany). Before silanization, the glass slides $(26 \times 76 \mathrm{~mm}$, Thermo Scientific, Braunschweig, Germany) were cleaned by sonification with ultrasound frequency $37 \mathrm{kHz}$ (Elmasonic $\mathrm{S} 60 \mathrm{H}$, Singen Germany) in 2\% detergent solution (Mucasol, Schuelke, Wertheim, Main, Germany) for $30 \mathrm{~min}$, rinsed with distilled water, sonicated for $5 \mathrm{~min}$ in acetone and airdried. Then the glass slides were sonicated in a $2 \%$ solution of proper aminosilane in toluene for $1 \mathrm{~h}$. The slides were baked in an oven at $120^{\circ} \mathrm{C}$ for $1 \mathrm{~h}$. Finally, the glass slides were rinsed for $5 \mathrm{~min}$ in each of the following: toluene, methanol-toluene (1:1), and methanol in an ultrasonic bath and airdried.

\section{Contact angle measurement}

Measurements of the static contact angle (q) were done using the DSA 100E auto goniometer (Krüss, Hamburg, Germany). The $3 \mathrm{~mL}$ sessile drops of water were used for the measurements, and the tangent angle to the drop at its intersection with the slide surface was assessed. In order to determine advancing contact angles, a minimum of eight independent measurements were performed, and results were presented as means \pm standard deviation.

\section{Assessment of antibody binding to the silanized surface \\ Antibody selection}

To evaluate the potential of the silanized glass surface to bind antibodies, the immunofluorescent reaction was used. To proceed with the reaction, the anti-EpCAM antibody was selected. The choice was made based on the researchers' future plan to catch EpCAM-positive cells. The HEA125 antibody was customized and provided by Miltenyi Biotech (Bergisch Gladbach, Germany). 


\section{Immunofluorescent reaction}

The immunofluorescent reaction was conducted as follows. Anti-EpCAM HEA125 antibody was diluted in phosphate-buffered saline (PBS) to obtain two working concentrations of $25 \mu \mathrm{g} / \mathrm{mL}$ and $5 \mu \mathrm{g} / \mathrm{mL}$. Each of three types of silanized glass surfaces $(\mathrm{A}, \mathrm{AE}$, and AEE) and non-modified glass surface were divided into three areas covered with both antibody concentrations and solely PBS used as a negative control. Tested glass surfaces were incubated with the above dilutions for $1 \mathrm{~h}$ at RT. Afterward, the unbound antibody was removed by washing with PBS repeated three times. Then $3 \%$ bovine serum albumin (BSA) was applied to glass slides for 30 min to block unspecific binding. After removing BSA solution, $2 \mu \mathrm{g} /$ $\mathrm{mL}$ of secondary FITC-labeled goat anti-mouse antibody (MFP488, goat anti-mouse IgG, MoBiTec, Goettingen, Germany) in PBS was used to detect primary anty-EpCAM antibody. Finally, after $1 \mathrm{~h}$ incubation, the unbound antibody was removed from glass slides by three washes with PBS, and glass slides were sealed with an antifade mounting medium (Vectashield, Vector Laboratories Inc., Burlingame, USA).

\section{Fluorescence intensity measurement}

The affinity of anti-EpCAM antibodies to tested silanized surfaces was evaluated by measuring the medium fluorescence intensity (MFI) in each analyzed area. For this purpose confocal fluorescent microscope (Olympus Fluoview i10) with appropriate software was used. To assess the MFI values, 5 regions of interest (ROIs) were selected on each area and photographed. The obtained values were presented in the form of histograms. Each studied glass slide was tested twice.

\section{Statistical analysis}

Statistical analysis was performed using the STATISTICA 13.3 PL software (StatSoft, Tulsa, OK, USA). The data were analyzed for statistically significant differences using one-way ANOVA with a post-hoc test. A p-value $<0.05$ was considered to be significant.

\section{Results \\ Contact angle}

Silanization with A, AE, and AEE significantly increased contact angles, confirming successful modification of the glass slide surface. However, the contact angles differed depending on the number of amino groups and length of the amino-functional chain of the employed silane ( $p<0.01$, Fig. 1). The results suggest that an increase in the number of amino groups decreases contact angle. Contact angle measurement was repeated for silanized glass slides following the incubation with $25 \mu \mathrm{g} / \mathrm{mL}$ and $5 \mu \mathrm{g} / \mathrm{mL}$ of anti-EpCAM antibody. In the case of A, we observed an increase of contact angle after incubation with $25 \mathrm{mg} /$ $\mathrm{mL}$ of antibodies and a decrease of contact angle after incubation with $5 \mathrm{mg} / \mathrm{mL}$ of antibodies. However, in case of AE and AEE, we observed reversed tendency a decrease of contact angles after incubation with 25 $\mathrm{mg} / \mathrm{mL}$ of antibodies and an increase of contact angles after incubation with $5 \mathrm{mg} / \mathrm{mL}$ of antibodies. Furthermore, the decrease for AEE was significantly higher $(\mathrm{p}$ $<0.01$, Fig. 1). Changes in the contact angles before and after the antibody's immobilization suggest that it was successfully attached to the tested surfaces. It also indicates that the length and flexibility of the amino-functional chain and the number of amino groups play a significant role in the efficiency of immobilization. This subject requires further studies.

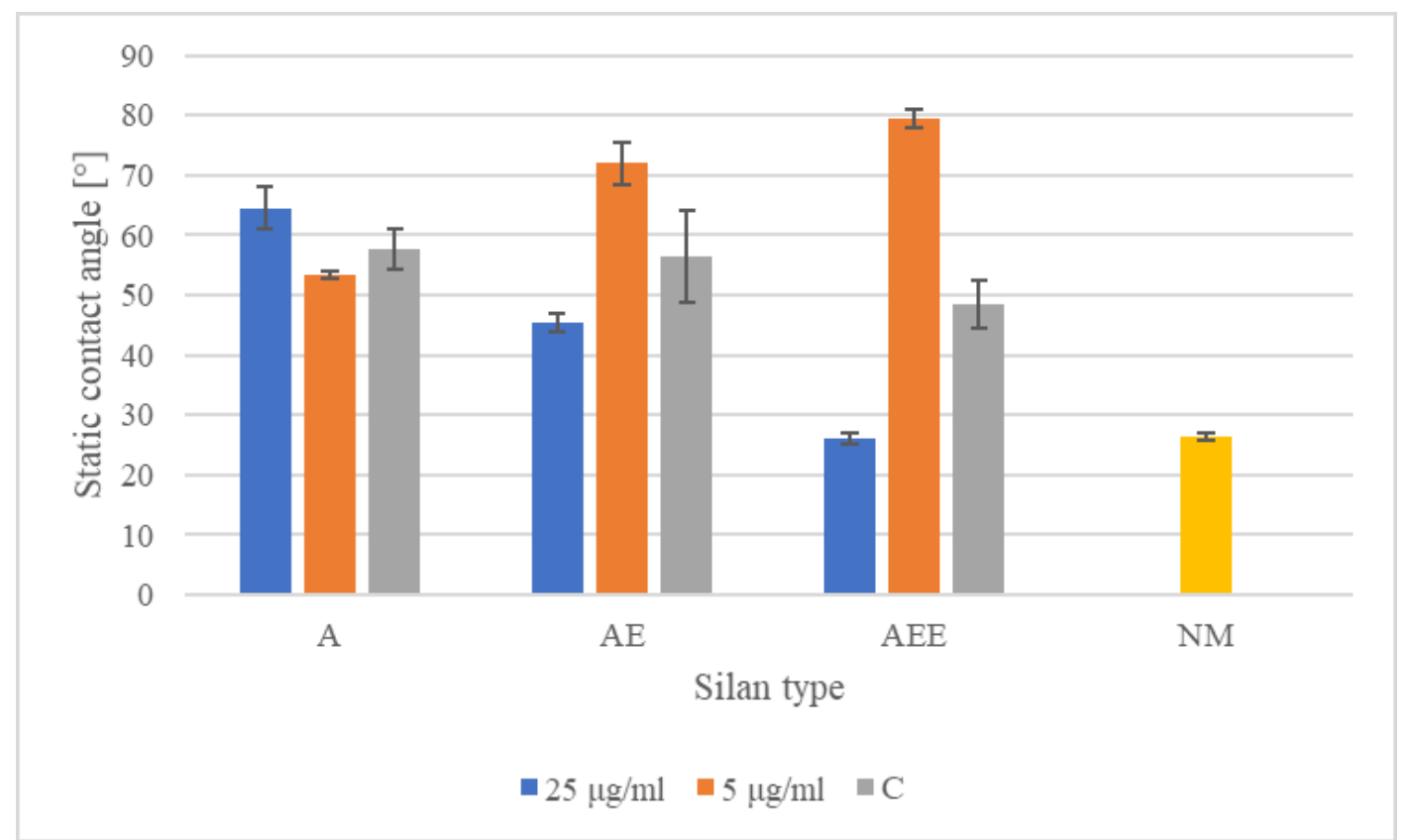

FIGURE 1 Comparison of average values of contact angles for tested glass slides surfaces: silanized with A, AE, AEE, and non-modified (NM) for areas covered with anti-EpCAM antibody in PBS (25 $\mu \mathrm{g} / \mathrm{mL}$ and $5 \mu \mathrm{g} / \mathrm{mL})$, and for control area covered with PBS (C) 


\section{Fluorescence intensity}

Analysis of fluorescence intensity also showed differences between tested silanes. All of them presented significantly higher fluorescence intensity in areas with immobilized antibodies compared to the control area ( $<<0.01$, Fig. 2$)$. It proved that the intensity of fluorescence reflects the amount of immobilized antibodies. The results suggest that the AE silane binds the anti-EpCAM antibody most efficiently. It should also be noted that increasing the antibody concentration from $5 \mu \mathrm{g} / \mathrm{mL}$ to $25 \mu \mathrm{g} / \mathrm{mL}$ resulted in higher fluorescence intensity for compounds $\mathrm{A}$ and AE. For the AEE compound, no such relationship was found.

Most importantly, it was found that all presented silanes had the ability to enhance antibody's binding efficiently since fluorescence intensity was significantly higher ( $p<0.01$, Fig. 2 ) in comparison to antibody-immobilized non-modified glass slides.

\section{Discussion}

The constant development of immunodiagnostics aims to find the most specific, selective, and sensitive assays based on capturing antibodies. This brings the need for efficient and oriented antibodies' immobilization on solid carriers. In the case of the most commonly used passive adsorption of antibodies, their proper orientation can be assured by pre-modification of the surface [22]. The promising option is silanization of the surface, helping to properly accommodate target antibodies and retain their binding activity [23].

In our research, silanization of glass slides was performed using three previously mentioned amino-functional trialkoxysilanes - A, AE, and AEE. The immunofluorescent reaction was used to evaluate the potential of the silanized glass surface to bind anti-EpCAM antibodies. The affinity of selected anti-EpCAM HEA125 antibodies labeled with fluorochrome to tested silanized surfaces was evaluated by measuring the mean fluorescence intensity (MFI) in each analyzed area.

Our results indicate that the presented silanes can effectively bind antibodies as higher fluorescence intensity was noticed in the case of silane-coated glass slides compared to unmodified ones. The differences in the contact angles also confirmed this result. In the case of silane $\mathrm{A}$, the fluorescence intensity reflected the amount of bound antibodies. However, there was no such a relation in the case of the silanes AE and AEE. This can be explained by the fact that a higher concentration of antibodies can affect the packing density. It may also lead to the steric hindrance in antigen binding [24-26]. The length and flexibility of amino-functional chains and the number of amino groups are crucial for the effective immobilization of antibodies. Equally, the physical and chemical stability of the immobilized antibodies still causes many problems because their binding activity can decrease after immobilization due to their random orientation [24]. That is why an appropriate immobilization method is vital to maintain the highest functionality of antibodies by site-specific orientation.

In the current study, we applied the passive adsorption of antibodies on silanized glass surfaces. In this approach, either antibodies or surfaces are subjected to prerequisite modification. Despite the simplicity of this method, it carries the risk of random antibodies' orientation driven by multiple

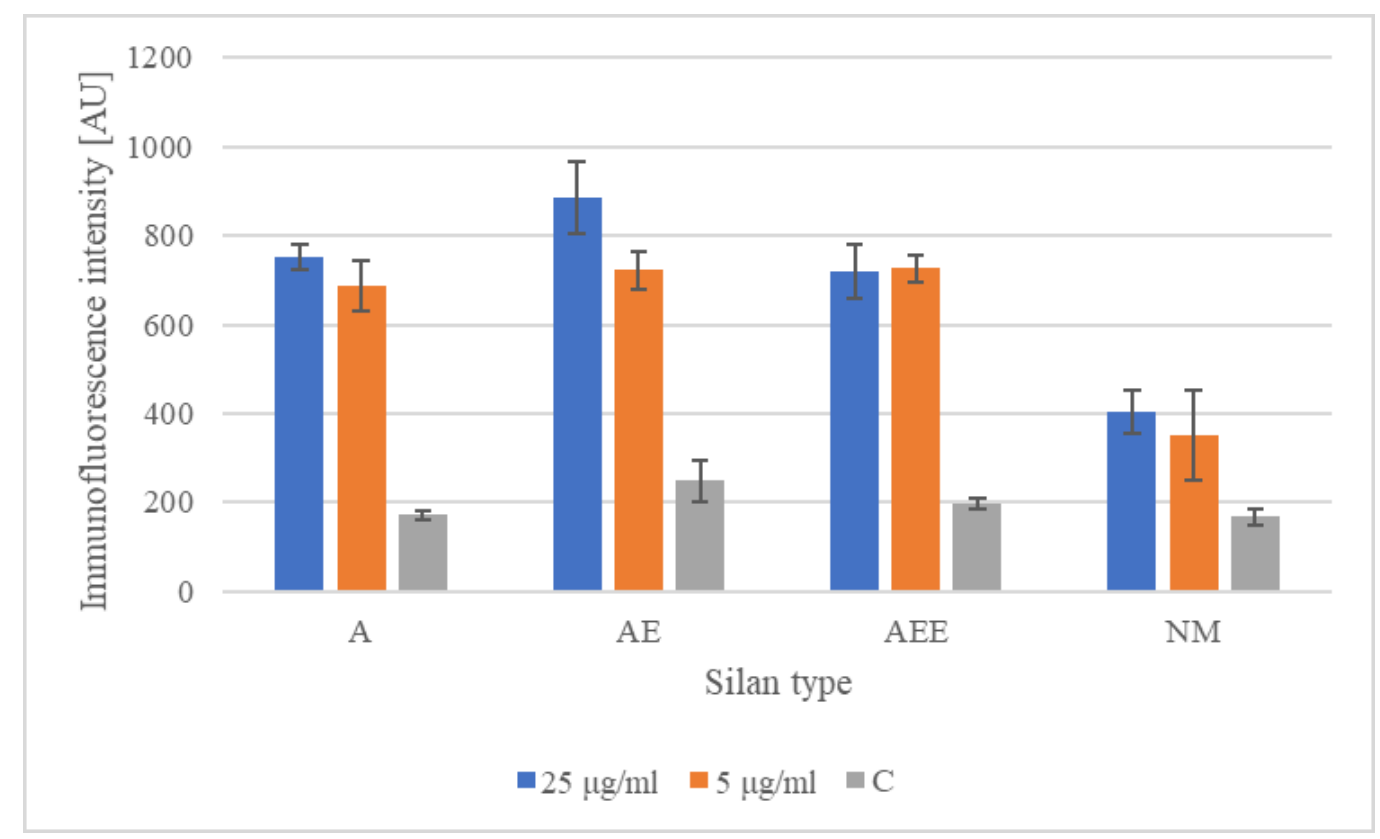

FIGURE 2 Immunofluorescence intensity of tested glass slides surfaces: silanized with A, AE, AEE, and non-modified (NM) for areas covered with anti-EpCAM antibody in PBS (25 $\mu \mathrm{g} / \mathrm{mL}$ and $5 \mu \mathrm{g} / \mathrm{mL})$, and for control area covered with PBS (C) 
non-covalent interactions, including van der Waals, electrostatic, and hydrophobic, strongly dependent on the $\mathrm{pH}$ and ionic strength [27]. To improve the oriented antibodies' binding, both - antibodies and surfaces can be subjected to various modifications employing, e.g., electric field [28] and UV light [29]. It was already observed that the charged surface helped obtain the better orientation of passively immobilized IgG1 and IgG2a antibodies. It was assumed that a low charge density of the surface and high ionic strength of the solution resulted in numerous orientations, caused by van der Waals interactions. Conversely, a high charge density and low ionic strength, respectively, caused certain immobilization patterns through solely positively or negatively charged antibody regions [30]. Another worth mentioning aspect is the influence of solution on already immobilized antibodies. The presence of other, highly charged, or more hydrophobic proteins can result in the elution of bound target antibodies [31].

All issues mentioned above can lead to random and inefficient immobilization of antibodies. Thus, sequentially can impair their affinity toward respective antigens and thus biomolecules. Various strategies of antibodies' immobilization are applied to solve this problem, including covalent or specifically directed non-covalent binding [32]. Among them, modification of tested surfaces with silanes is promising, since antibodies can be immobilized on the modified surfaces by forming a covalent bond between their functional and complementary silane coupling groups [21]. It was also found that aminosilanes could bind the antibodies in the correct orientation and preserve their binding activity [23].

The results of our research indicate silanes as effective antibody immobilizers. This could consequently lead to the development of silane-based devices devoted to isolation of rare cells, including CTCs. However, this requires further studies using selected cancer cells to confirm the effectiveness of selected silanes.

Commonly used 3-Aminopropyltriethoxysilane (A) has already been used for surface functionalization [18]. In the study by Sterzynska and Budna et al. A, A + 3-(octafluoropentyloxy)-propyltriethoxysilane and A + n-butyltrimethoxysilane were used. Like in our study, glass plates as platforms for the silane coating and anti-EpCAM antibody were used. Additionally, the authors analyzed the ability of immobilized anti-EpCAM antibodies to capture cancer cells. It was concluded that modification of A with non-reactive silanes improved the efficiency of antibody capture. It justifies the subsequent attempts to find other silanes that may be even more effective in antibody binding.

Immobilization of antibodies has several applications in science, including diagnostic methods, but might also be used in devices applied in e.g., pharmacy and proteomics as biosensors [33]. The litera- ture shows the studies aiming to select the best immobilizer for capturing specific biomolecules and cells. Immobilizers are frequently compared in various studies. Some researchers utilize substances from the group of silanes, as well as other platforms, including gold-plated. In a study by Cetin et al., two techniques of gold surface modification were presented to immobilize anti-EpCAM antibodies to detect CTCs. The authors used for modification of self-assembled monolayers (SAMs) [16], which reduce non-specific binding of molecules regard to pure metal [34]. The antibodies were bound in two ways - by covalent bonds and by a bioaffinity interaction. In the second case, streptavidin and biotinylated antibodies were used. The results indicated that the presence of long aromatic chains containing alkanethiols promoted better cell-uptaking [16].

Not only silanes are applied as immobilizers. In the study by Cohn et al., cholesteryl succinyl silane (CSS) fibers, hydrophobic polycaprolactone (PCL) fibers, and hydrophilic plasma-treated PCL fibers were coated on silicon chips in order to improve anti-CD20 antibody binding. Immobilization of antibodies via lipid fibers improved the preservation of their function. Among the tested solutions, the CSS with anti-CD20 antibodies captured the higher number of B-cell lymphoma cells [33].

In the study by Foerster et al., a method of immobilizing recombinant antibody fragments ( $\mathrm{scFv}$ ) on 316L stainless steel to improve the growth of human endothelial progenitor cells (EPCs) was presented. This solution improved their viability on implanted stents, which was beneficial for cardiovascular applications. Moreover, the amino-functional and antibody-coated surfaces were nontoxic to these cells [35].

The promising results of our current research encourage to validate them evaluating the cells' uptake by the tested surface. In the long term, such a study could contribute to the development of in vivo methods similar to the those like GILUPI CellCollector (GILUPI GmbH). This stainless steel medical wire is covered with a gold and polymer layer (hydrogel), where anti-EpCAM antibodies are bound covalently $[8,36]$. The silane-based approach might also be helpful for the improvement of antibody-based immunoassays, e.g., ELISA. However, all the above require highly specific and selective uptake of cells or biomolecules from very complex biological samples, which underlies the need for efficient and oriented antibody immobilization [22].

Furthermore, equally important in successful antibodies' immobilization are antibodies by themselves. This regards especially their molecular mass, flexibility, stability, and accessibility of their binding regions [37]. Thus, it is recommended to extend future research and evaluate the correlation between immobilizer and various antibodies, including recombinant ones. 


\section{Conclusions}

Successful immobilization of antibodies has a number of promising applications, including detection of CTCs in different types of cancer. The problem of random antibodies' orientation can be solved by modification of tested surfaces with silanes. We found that $\mathrm{A}, \mathrm{AE}$ and $\mathrm{AEE}$ aminosilanes were effective antibody immobilizers, helping to accommodate them in the correct orientation and thus preserving their binding activity. This observation can improve the development of silane-based devices devoted to CTCs isolation. However, the usefulness of selected silanes needs to be confirmed by further studies using cancer cells.

\section{Ethical approval}

The conducted research is not related to either human or animal use.

\section{Acknowledgements}

This research was supported by the ERA-NET TRANSCAN/01/2018 "PROLIPSY" project from The National Centre for Research and Development.

\section{Corresponding author}

Joanna Budna-Tukan, Department of Histology and Embryology, Poznań University of Medical Sciences, Święcickiego 6 St., 61-701 Poznań, Poland, tel. +48 618546428, e-mail: jbudna@ump.edu.pl.

\section{Conflict of interests statement}

The authors declare they have no conflict of interest.

\section{References}

1. Alix-Panabières $\mathrm{C}$, Schwarzenbach $\mathrm{H}$, Pantel K. Circulating tumor cells and circulating tumor DNA. Annu Rev Med. 2012;63:199-215; DOI:10.1146/annurev-med-062310-094219.

2. Paoletti C, Hayes DF. Circulating Tumor Cells. Adv Exp Med Biol. 2016;882:235-58; DOI:10.1007/978-3-319-22909-6_10.

3. Paterlini-Brechot P, Benali NL. Circulating tumor cells (CTC) detection: clinical impact and future directions. Cancer Lett. 2007;253(2):180204; DOI:10.1016/j.canlet.2006.12.014.

4. LianidouES, MarkouA,StratiA.The RoleofCTCsasTumorBiomarkers. Adv Exp Med Biol. 2015;867:341-67; DOI:10.1007/978-94-017-7215-0_21.

5. Dianat-Moghadam H, Azizi M, Eslami-S Z, Cortés-Hernández LE, Heidarifard M, Nouri M, i in. The Role of Circulating Tumor Cells in the Metastatic Cascade: Biology, Technical Challenges, and Clinical Relevance. Cancers. 2020;12(4); DOI:10.3390/cancers12040867.

6. Maly V, Maly O, Kolostova K, Bobek V. Circulating Tumor Cells in Diagnosis and Treatment of Lung Cancer. In Vivo. 2019;33(4):1027-37, DOI:10.21873/invivo.11571.

7. Dizdar L, Fluegen G, van Dalum G, Honisch E, Neves RP, Niederacher D, in. Detection of circulating tumor cells in colorectal cancer patients using the GILUPI CellCollector: results from a prospective, single-center study. Mol Oncol. 2019;13(7):1548-58; DOI:10.1002/1878-0261.12507.

8. Gorges TM, Penkalla N, Schalk T, Joosse SA, Riethdorf S, Tucholski J, i in. Enumeration and Molecular Characterization of Tumor Cells in Lung Cancer Patients Using a Novel In Vivo Device for Capturing Circulating Tumor Cells. Clin Cancer Res Off J Am Assoc Cancer Res. 2016;22(9):2197-206; DOI:10.1158/1078-0432.CCR-15-1416.

9. Pantel K, Hille C, Scher HI. Circulating Tumor Cells in Prostate Cancer: From Discovery to Clinical Utility. Clin Chem. 2019;65(1):87-99; DOI:10.1373/clinchem.2018.287102.

10. Poulet G, Massias J, Taly V. Liquid Biopsy: General Concepts. Acta Cytol. 2019;63(6):449-55; DOI:10.1159/000499337.

11. Habli Z, AlChamaa W, Saab R, Kadara H, Khraiche ML. Circulating Tumor Cell Detection Technologies and Clinical Utility: Challenges and Opportunities. Cancers. 2020;12(7); DOI:10.3390/cancers12071930.

12. Saucedo-Zeni N, Mewes S, Niestroj R, Gasiorowski L, Murawa D, Nowaczyk P, i in. A novel method for the in vivo isolation of circulating tumor cells from peripheral blood of cancer patients using a functionalized and structured medical wire. Int J Oncol. 2012;41(4):1241-50; DOI:10.3892/ijo.2012.1557.
13. Shahneh FZ. Sensitive antibody-based CTCs detection from peripheral blood. Hum Antibodies. 2013;22(1-2):51-4; DOI:10.3233/HAB-130270.

14. Hvichia GE, Parveen Z, Wagner C, Janning M, Quidde J, Stein A, i in. A novel microfluidic platform for size and deformability based separation and the subsequent molecular characterization of viable circulating tumor cells. Int J Cancer. 2016;138(12):2894-904; DOI:10.1002/ijc.30007.

15. Gires O, Pan M, Schinke H, Canis M, Baeuerle PA. Expression and function of epithelial cell adhesion molecule EpCAM: where are we after 40 years? Cancer Metastasis Rev. 2020;39(3):969-87; DOI:10.1007/ s10555-020-09898-3.

16. Cetin D, Okan M, Bat E, Kulah H. A comparative study on EpCAM antibody immobilization on gold surfaces and microfluidic channels for the detection of circulating tumor cells. Colloids Surf B Biointerfaces. 2020;188:110808; DOI:10.1016/j.colsurfb.2020.110808.

17. Tao L, Zhang K, Sun Y, Jin B, Zhang Z, Yang K. Anti-epithelial cell adhesion molecule monoclonal antibody conjugated fluorescent nanoparticle biosensor for sensitive detection of colon cancer cells. Biosens Bioelectron 2012;35(1):186-92; DOI:10.1016/j.bios.2012.02.044.

18. Vashist SK, Lam E, Hrapovic S, Male KB, Luong JHT. Immobilization of antibodies and enzymes on 3-aminopropyltriethoxysilane-functionalized bioanalytical platforms for biosensors and diagnostics. Chem Rev. 2014;114(21):11083-130; DOI:10.1021/cr5000943.

19. Piascik JR, Wolter SD, Stoner BR. Development of a novel surface modification for improved bonding to zirconia. Dent Mater Off Publ Acad Dent Mater. 2011;27(5):e99-105; DOI:10.1016/j.dental.2011.01.005.

20. Vansant EF, Voort PVD, Vrancken KC. Characterization and Chemical Modification of the Silica Surface. Elsevier. 1995. 573 s.

21. MacBeath G, Schreiber SL. Printing proteins as microarrays for high-throughput function determination. Science. 2000;289(5485):1760-3; DOI: 10.1126/science.289.5485.1760.

22. Shen M, Rusling J, Dixit CK. Site-selective orientated immobilization of antibodies and conjugates for immunodiagnostics development. Methods San Diego Calif. 2017;116:95-111; DOI:10.1016/j. ymeth.2016.11.010.

23. Couston RG, Skoda MW, Uddin S, van der Walle CF. Adsorption behavior of a human monoclonal antibody at hydrophilic and hydrophobic surfaces. mAbs. 2013;5(1):126-39; DOI:10.4161/mabs.22522.

24. Sterzynska K, Budna J, Frydrych-Tomczak E, Hreczycho G, Malinska A, Maciejewski H, i in. Silane-modified surfaces in specific antibody-mediated cell recognition. Folia Histochem Cytobiol. 2014;52(3):250-5; DOI:10.5603/FHC.2014.0029.

25. Xu H, Zhao X, Grant C, Lu JR, Williams DE, Penfold J. Orientation of a monoclonal antibody adsorbed at the solid/solution interface: a combined study using atomic force microscopy and neutron reflectivity. Langmuir ACS J Surf Colloids. 2006;22(14):6313-20; DOI:10.1021/ la 0532454 .

26. Xu H, Zhao X, Lu JR, Williams DE. Relationship between the structural conformation of monoclonal antibody layers and antigen binding capacity. Biomacromolecules. 2007;8(8):2422-8; DOI:10.1021/bm070297u.

27. Zhao X, Pan F, Garcia-Gancedo L, Flewitt AJ, Ashley GM, Luo J, i in. Interfacial recognition of human prostate-specific antigen by immobilized monoclonal antibody: effects of solution conditions and surface chemistry. J R Soc Interface. 2012;9(75):2457-67; DOI:10.1098/ rsif.2012.0148.

28. Emaminejad S, Javanmard M, Gupta C, Chang S, Davis RW, Howe RT. Tunable control of antibody immobilization using electric field. Proc Natl Acad Sci U S A. 2015;112(7):1995-9; DOI:10.1073/pnas.1424592112.

29. Della Ventura B, Schiavo L, Altucci C, Esposito R, Velotta R. Light assisted antibody immobilization for bio-sensing. Biomed Opt Express. 2011;2(11):3223-31; DOI:10.1364/BOE.2.003223.

30. Zhou J, Tsao H-K, Sheng Y-J, Jiang S. Monte Carlo simulations of antibody adsorption and orientation on charged surfaces. J Chem Phys. 2004;121(2):1050-7; DOI:10.1063/1.1757434.

31. Harmsen MM, Fijten HPD. Improved functional immobilization of llama single-domain antibody fragments to polystyrene surfaces using small peptides. J Immunoassay Immunochem. 2012;33(3):234-51; D0I:10.1 080/15321819.2011.634473.

32. Holstein CA, Chevalier A, Bennett S, Anderson CE, Keniston K, Olsen C $\mathrm{i}$ in. Immobilizing affinity proteins to nitrocellulose: a toolbox for paper-based assay developers. Anal Bioanal Chem. 2016;408(5):1335-46; DOI:10.1007/s00216-015-9052-0.

33. Cohn C, Leung SL, Zha Z, Crosby J, Teng W, Wu X. Comparative study of antibody immobilization mediated by lipid and polymer fibers. Colloids Surf B Biointerfaces. 2015;134:1-7; DOI:10.1016/j. colsurfb.2015.06.021.

34. Baldrich E, Laczka O, del Campo FJ, Muñoz FX. Self-assembled monolayers as a base for immunofunctionalisation: unequal performance for protein and bacteria detection. Anal Bioanal Chem. 2008;390(6):155762; DOI:10.1007/s00216-008-1887-1. 
35. Foerster A, Hołowacz I, Sunil Kumar GB, Anandakumar S, Wall JG, Wawrzyńska M, i in. Stainless steel surface functionalization for immobilization of antibody fragments for cardiovascular applications. J Biomed Mater Res A. 2016;104(4):821-32; DOI:10.1002/jbm.a.35616.

36. Theil G, Fischer K, Weber E, Medek R, Hoda R, Lücke K, i in. The Use of a New CellCollector to Isolate Circulating Tumor Cells from the Blood of Patients with Different Stages of Prostate Cancer and Clinical Outcomes - A Proof-of-Concept Study. PloS One. 2016;11(8):e0158354; DOI:10.1371/journal.pone.0158354.

37. Sharma S, Byrne H, O'Kennedy RJ. Antibodies and antibody-derived analytical biosensors. Essays Biochem. 2016;60(1):9-18; DOI:10.1042/ EBC20150002. 